\section{Recurrent cutaneous abscesses in two Italian family members}

\author{
Carmen Cantisani, ' Antonio G. Richetta,' \\ Andrea Bitonti,' Pietro Curatolo,' \\ Gianfranco Ferretti, ${ }^{2}$ Carlo Mattozzi, \\ Melis Luca, ' Emidio Silvestri, ${ }^{1}$ \\ Stefano Calvieri ${ }^{1}$ \\ 'Department of Oncoematology- \\ Dermatology and Plastic Surgery; \\ 2Department of Tropical Medicine, \\ Azienda Policlinico Umberto I, University \\ "Sapienza" of Rome, Italy
}

\begin{abstract}
Environmental mycobacteria are the causative factors of an increasing number of infections worldwide. Cutaneous infections as a result of such mycobacteria are often misdiagnosed, and their treatment is difficult since they can show in vivo and in vitro multidrug resistance. Absence of pathognomonic clinical signs and variable histological findings often delay diagnosis. We report a case of localized recurrent soft tissue swelling by Mycobacterium marinum in 2 members of the same family. The cases are being reported for their uncommon clinical presentation and the associated etiological agent. Patients recovered completely following therapy with rifampicin $600 \mathrm{mg}$ plus isoniazide $300 \mathrm{mg}$ daily for 45 days.
\end{abstract}

\section{Introduction}

Skin and soft tissue infections caused by non-tuberculous mycobacteria (NTM), which used to be considered unusual, have become more frequent in recent times. ${ }^{1}$ Atypical mycobacteria are environmental saprophytes, which may occasionally enter human skin through injuries and cause localized infection. Papules, nodules, plaques, ulcers and panniculitis-like lesions are common manifestations. ${ }^{2,3}$ Disseminated infection occurs in immunocompromised patients. This kind of cutaneous infection is not frequently seen in humans and its diagnosis can be missed unless there is a strong clinical suspicion coupled with microbiological confirmation.

\section{Case Reports}

A 52-year old healthy upper middle class, Italian male and his 21-year old daughter presented in May 2008 with a history of swelling on of the father's face and neck, and on the daughter's buttocks and legs. The swellings had started approximately three months previously, had gradually increased in size, associated with moderate pain. They were of insidious onset without any preceding history of trauma or infection. Both subjects reported that they were swimmers and that they used to travel to Egypt in the summer. On physical examination, the daughter had purple indurated subcutaneous nodules and plaques were observed on her right buttock. The father had subcutaneous granulomas on the lateral left side of his neck that had also ulcerated (Figure 1). Both patients reported intermittent discharge from the lesions and associated local non-tender lymphadenitis. There was a local rise of temperature without fever. Systemic examination of patients did not reveal any abnormality. Diagnostic aspiration from the swelling had been performed approximately 20 days previously after which the swelling had subsided. The cytological findings of the aspirate were inconclusive and the swelling recurred within the following few days which led to the patients returning for a repeat evaluation. Laboratory evaluation, including WBC count, chain protein reaction (CPR) and Antistreptolysin 0 titre (AS(L)0 titre (ASOT), showed no increase in values. Rheumatoid factor and HIV (performed with patient consent) were negative. Liver, renal function tests and a chest X-ray were within normal limits. Needle aspiration from the swelling was repeated and subjected to cytological and microbiological evaluations which showed cream colonies that turned yellow when exposed to light. A repeated skin biopsy specimen of the abscess wall showed an acute-on-chronic infection with edematous granulation tissue and some foreign-body giant cells. A focus of caseation necrosis was also noted. A Ziehl-Neelson stain showed acid fast bacilli in the necrotizing granuloma. Molecular diagnosis can identify species of mycobacteria polymerase chain reaction-restriction fragment of the $h s p 65$ gene. After eight weeks, the culture grew Mycobacterium marinum. Patients were treated successfully with rifampicin $600 \mathrm{mg}$ plus isoniazid $300 \mathrm{mg}$. After two months of treatment, the lesions healed completely. At 1-year follow up, there was no recurrence of the disease.
Correspondence: Carmen Cantisani, Department of Oncohematology-Dermatology and Plastic Surgery, Azienda Policlinico Umberto I, University "Sapienza", viale del Policlinico 155, 00167 Rome, Italy. E-mail: carmencantisanister@gmail.com; carmen.cantisani@uniromal.it

Key words: cutaneous infection, mycobacteria, treatment, immunocompetent host.

Acknowledgments: we would like to thank Dr Anna Spataro and Mrs Sonia Tofani, Department of Dermatology and Plastic Surgery, University Sapienza of Rome, Italy for their precious help.

Received for publication: 17 June 2010.

Revision received: 15 July 2010.

Accepted for publication: 15 July 2010 .

This work is licensed under a Creative Commons Attribution 3.0 License (by-nc 3.0).

(C) Copyright C Cantisani et al., 2010

Licensee PAGEPress, Italy

Infectious Disease Reports 2010; 2:e11

doi:10.4081/idr.2010.e11

\section{Discussion}

Mycobacterium has 95 well-characterized species. ${ }^{4}$ Two well-known species, $M$. tuberculosis and $M$. leprae, have been known for centuries to cause immense human suffering. The pathogenic potential of most other mycobacteria, present in the environment as saprophytes, has been recognized since the beginning of the $20^{\text {th }}$ century. Mycobacterium marinum belongs to Runyon Group 1 photochromogenic non-tuberculous mycobacteria. It is a waterborne mycobacterium that commonly infects fish and amphibians all over the world.

Infection in humans occasionally occur, especially in a country like Italy. Categories at risk are home-aquarium enthusiasts, swimmers, aquarium staff, marine-life handlers, anglers, and oyster workers. In most cases, they present as a granulomatous infection localized in the skin, typically following minor trauma and other surgical procedures. The usual presentation is trauma to the skin in non-chlorinated water or salt water, and after
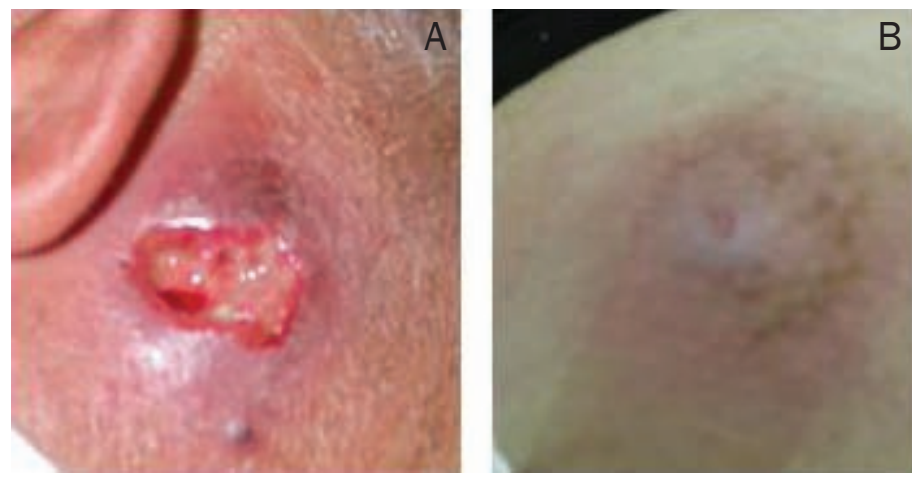
ed nodules on the neck. (B) A painful abscess with associated erythema and edema of the right buttock. 
about two weeks of inoculation it will develop into a localized papulonodular lesion which eventually ulcerates. The clinical syndrome is variable, the pathology is non-specific and culture is needed for definite diagnosis. Infections sometimes heal spontaneously, but drug treatment is usually necessary for several months in order to cure the infection. Treatment is often delayed because of lack of diagnostic suspicion or cost. Today, it remains an important differential diagnosis for papulonodular lesion which eventually ulcerates. Skin disorders are common in travelers. Knowledge of the relative frequency of post-travel related skin disorders, including their geographical and demographic risk factors, will allow for effective pre-travel counseling, as well as improve post-travel diagnosis and therapeutic intervention. In our patients, suspicion was aroused because they were swimmers who used to travel to Egypt during the summer and had reported contact with aquatic animals, although they could not recall how many months after the trip the lesions appeared. Clinicians seeing patients post-travel should be alert to travel-related skin diseases. ${ }^{6}$ To prevent and manage skin-related morbidity during travel, international travelers should avoid direct contact with sand, soil, and aquatic animals. Sometimes lesions are aspecific and appear many months later so it is important to keep record of any traveling and seek immediate medical care.

With the increased numbers of immunocompromised patients, it is likely that we will see a rising trend in these infections. A high index of suspicion is the key to diagnosis and appropriate samples should be sent for mycobacterial culture before the use of antibiotics.

\section{References}

1. Streit M, Bregenzer T, Heinzer I. Cutaneous infections due to atypical mycobacteria. Hautarzt 2008;59:59-70.
2. Bhambri S, Bhambri A, Del Rosso JQ. Atypical mycobacterial cutaneous infections. Dermatol Clin 2009;27:63-73.

3. Song H, Lee H, Choi G, et al. Cutaneous nontuberculous mycobacterial infection: a clinicopathological study of 7 cases. Am J Dermatopathol 2009;31:227-31.

4. Van Ingen J, de Zwaan R, Dekhuijzen R, et al. Region of difference 1 in nontuberculous Mycobacterium species adds a phylogenetic and taxonomical character. J Bacteriol 2009;191:5865-7.

5. Cheung JP, Fung B, Wong SS, et al. Review article: mycobacterium marinum infection of the hand and wrist. J Orthop Surg (Hong Kong) 2010;18:98-103.

6. Petrini B. Mycobacterium marinum: ubiquitous agent of waterborne granulomatous skin infections. Eur J Clin Microbiol Infect Dis 2006;25:609-13. 